\title{
Real and complex analytic sets. The relevance of Segre varieties
}

\author{
KLAS Diederich AND EMmanUel MAZZILli
}

\begin{abstract}
Let $X \subset \mathbb{C}^{n}$ be a closed real-analytic subset and put
$\mathcal{A}:=\left\{z \in X \mid \exists A \subset X\right.$, germ of a complex-analytic set, $\left.z \in A, \operatorname{dim}_{z} A>0\right\}$

This article deals with the question of the structure of $\mathcal{A}$. In the main result a natural proof is given for the fact, that $\mathcal{A}$ always is closed. As a main tool an interesting relation between complex analytic subsets of $X$ of positive dimension and the Segre varieties of $X$ is proved and exploited.
\end{abstract}

Mathematics Subject Classification (2000): 32B10 (primary); 32C07 (secondary).

\section{Introduction}

If $X$ is a closed real-analytic set in an open subset $U \subset \mathbb{C}^{n}, n>1$, two questions may be asked:

1. What is a good notion of a complexification of $X$, more precisely, what is the smallest germ of a complex analytic set along $X$ in $\mathbb{C}^{n}$, containing $X$ ?

2. Which complex-analytic sets are contained in $X$ and how can they be found?

This article deals with the second question. More precisely, we consider the following situation:

Let $X \subset U$ be as above. Put

$$
\mathcal{A}:=\left\{z \in X \mid \exists z \in A \subset X, A \text { germ of a complex-analytic set, } \operatorname{dim}_{z} A>0 .\right\}
$$

Our principal goal is to show

Theorem 1.1 (Main Result). The set $\mathcal{A}$ is closed.

In the algebraic case, the result has been proved in [8]. Also the case of realanalytic hypersurfaces has been announced in the literature. In [7] it is stated for pseudoconvex $\mathcal{C}^{\omega}$ compact hypersurfaces as a theorem. However, the proof in the article causes difficulties. Furthermore, the main result is mentioned by d'Angelo in 
[4]. As Theorem 2 on page 131 he states for a real-analytic smooth $\mathcal{C}^{\omega}$-hypersurface $H$ the following: if $x$ is a limit point of $\mathcal{A}$ then $H$ does not have finite type at $x$. (This result also is contained in [3].) This is correct. However, he then wants to show that through any point $x$ of infinite type on $H$ there is a complex-analytic set of positive dimension in $X$ (Theorem 4, page 134). His proof, however, seems to be incomplete, and so the closedness of $\mathcal{A}$ has to be proved differently. The proof given in this article seems to us very natural and direct. It works in great generality.

A major tool for the proof of the Main result is the following theorem, which was already proved by Diederich/Fornæss in [5]:

Theorem 1.2 (Globalization). Let $\gamma \subset X$ be a germ of a holomorphic curve, $0 \in$ $\gamma$. There is a positive radius $r>0$ which depends only on $X$, such that in the ball of radius $r$ around $0, B_{r}(0)$ there is a closed complex analytic set $A$ with $\gamma \subset A \subset X$.

Remark 1.3. The essential fact in this result is that the radius $r>0$ can be chosen independently of the choice of 0 and $\gamma$.

In [5] it is shown that an immediate consequence of this is:

Corollary 1.4. If $X$ is compact, then $\mathcal{A}=\emptyset$.

Due to Kohn's theory of subelliptic multipliers from [7] together with [5, Theorem 3 , page 374$]$ the following result on the $\bar{\partial}$-Neumann problem follows, which nowadays is well known:

Theorem 1.5. Let $\Omega \Subset \mathbb{C}^{n}$ be a pseudoconvex domain with smooth $\mathcal{C}^{\omega}$ boundary. Then subelliptic estimates hold for the $\bar{\partial}-$ Neumann problem on $\Omega$ in all degrees.

Remark 1.6. This result probably is the most important application of the study of $\mathcal{A}$.

The main idea of the proof here is the use of the fact that Segre varieties depend (anti)holomorphically on their base points. After a lot of technical work this will enable us to use the following uniform volume estimate of Diederich-Pinchuk from [6] in order to ensure that certain sequences of analytic subsets of $X$ converge themselves to analytic subsets of $X$ due to the famous theorem of Bishop (see [1] and also [2, Theorem 15.5]). It says:

Theorem 1.7 (Bishops' theorem). Let $A_{v} \subset U$ be a sequence of pure p-dimensional analytic subsets of a complex manifold $X$ converging to some set $A \subset X$ and such that for any compact subset $K \subset X$ there is a constant $M_{K} \geq 0$ with

$$
\operatorname{vol}_{2 p}\left(A_{v} \cap K\right) \leq M_{K}
$$

for all $v$. Then $A$ is also a pure p-dimensional analytic subset of $X$. 
The uniform volume estimate is:

Theorem 1.8 (Diederich-Pinchuk). Let $U \subset \mathbb{C}^{n}, V \subset \mathbb{C}^{m}$ be open sets and $g_{j}(z, w) \in \mathcal{O}(U \times V)$ for $j=1, \ldots, k$ with a positive integer $k \leq n$. For $w \in V$ put

$$
A_{w}:=\left\{z \in U: g_{j}(z, w)=0 \text { for } j=1, \ldots, k\right\}
$$

Let $E:=\left\{w \in V: \operatorname{dim}_{\mathbb{C}} A_{w}>n-k\right\}$. Then for any $\tilde{U} \subset \subset U, \tilde{V} \subset \subset V$ there exists a constant $c=c(\tilde{U}, \tilde{V})>0$ such that

$$
\operatorname{vol}_{2(n-k)}\left(A_{w} \cap \tilde{U}\right)<c
$$

for all $w \in \tilde{V} \backslash E$. In particular, we can extract from any sequence $\left(A_{w_{v}}\right), w_{v} \in$ $\tilde{V} \backslash E$, a subsequence converging in $U$ to an analytic subset $A$ of pure dimension $n-k$. (Notice that the sequence $\left(w_{v}\right)$ might converge to a point in $E$.)

The research of this article can be the starting point for the clarification of several other puzzling questions. We mention two questions which seem to be open except for very special cases:

\section{Question 1.9.}

1. Is $\mathcal{A}$ itself a real analytic set?

2. Let $0 \in X$ be a point of infinite d'Angelo type. Is there a complex analytic germ $A$ of positive dimension with $0 \in A \subset X$ ?

The structure of the article is the following: In Section 2 we will show the "Principal Lemma" showing already the essential role which will be played by Segre varieties in the whole article. Theorem 2 will be easily reproved in Section 3 using it. In Section 4 we apply, for the first time, the theorem of Diederich-Pinchuk in order to get an analytic family of complete intersections (see Lemma 4.1 ) to which we can apply in Section 5 the [6] theorem again in order to get a suitable sequence of analytic sets in $X$ with bounded volume, such that, finally, the Bishop theorem gives a good analytic limit set in $X$ passing through 0 .

ACKNOWLEDGEMENTS. The main part of the research of this article was discussed while the first author was a visitor at the Institute for Mathematical Research (FIM) of the ETH Zürich. He thanks this institution for the hopitality and the excellent working conditions given to him. The final version of the article was written while the first author was a visitor at the Max-Planck-Institut für Mathematik in Bonn. He thanks this institution for the very valuable invitation. 


\section{The Principal Lemma}

We may assume (see Remark 2.1 below) that $X \subset U$ can be written in $U$ as

$$
X=\{z \in U \mid \varrho(z)=0\}
$$

and let $V \Subset U$ be a relatively compact subset and $z_{0} \in V$ arbitrary.

Remark 2.1. Notice that any real-analytic set can be locally written as the zero set of one real-analytic function $\varrho$, independently of its dimension. Of course, here we do not assume that $d \varrho$ nowhere vanishes.

Then there is a radius $r>0$ such that the power series expansion in $z$ and $\bar{z}$ of $\varrho$ at the point $z_{0}$

$$
\varrho(z, \bar{z})=\sum_{\alpha, \beta \in \mathbb{N}^{n}} \frac{1}{\alpha ! \beta !} \partial^{\alpha} \bar{\partial}^{\beta} \varrho\left(z_{0}, \bar{z}_{0}\right)\left(z-z_{0}\right)^{\alpha}\left(\bar{z}-\bar{z}_{0}\right)^{\beta}
$$

is convergent on the polycylinder $\Delta_{r}^{n}\left(z_{0}\right)$ of radius $r$ around $z_{0}$. The complexification $\hat{\varrho}(z, w)$ is the function defined for

$$
(z, w) \in \hat{U}:=\bigcup_{z_{0} \in V}\left\{\Delta_{r}^{n}\left(z_{0}\right) \times \Delta_{r}^{n}\left(z_{0}\right)\right\} \subset \mathbb{C}^{2 n}
$$

by replacing in (2.2) $\bar{z}$ by the new independent variable $\bar{w}$. The function $\hat{\varrho}(z, w)$ is holomorphic in $z$ and antiholomorphic in $w$. With it we define for any point $z_{0} \in V$ :

Definition 2.2. The Segre variety of $X$ at $z_{0}$ is the closed complex hypersurface

$$
S_{z_{0}}:=\left\{z \in \Delta_{r}^{n}\left(z_{0}\right) \mid \hat{\varrho}\left(z, z_{0}\right)=0\right\} .
$$

Remark 2.3. Notice that, even if we assume that $\varrho(z) \not \equiv 0$, it might happen that $\hat{\varrho}\left(\cdot, z_{0}\right) \equiv 0$, since we do not assume that $d \varrho$ nowhere vanishes. Another consequence of this is that $S_{z_{0}}$ does not only depend on $X$, but also on the choice of $\varrho$, and there is no canonical choice for $\varrho$.

The family of Segre varieties given by a fixed $\varrho$ satisfies also in the more general situation considered here the same elementary properties as usual. One has:

Lemma 2.4. Let the points $z \in V$ and $w$ be chosen such that $(z, w) \in \hat{U}$. Then

(1) $z \in S_{w} \quad \Leftrightarrow \quad w \in S_{z}$;

(2) $z \in S_{z} \quad \Leftrightarrow \quad z \in X$.

The following is the Principal Lemma for our work and shows the importance of the Segre varieties:

Lemma 2.5 (Principal Lemma). Let $X, \varrho, r$ be chosen as above. Assume that $0 \in$ $X$. If $0 \in \gamma \subset X$ is a piece of a non-constant holomorphic disc with holomorphic parametrization $z(t)$ then

$$
\gamma \subset S_{0}(X):=\left\{z \in \Delta^{n}(0, r) \mid \hat{\varrho}(z, 0)=0\right\} .
$$


Proof. We reorder the Taylor series expansion of the function $\hat{\varrho}(z(t), z(t))$ around the point $(z(t), 0)$ a little bit and get

$$
\underbrace{\hat{\varrho}(z(t), \overline{z(t)})}_{\equiv 0, \text { since } \gamma \subset X}-\underbrace{\hat{\varrho}(z(t), 0)}_{\text {hol. in } t}=\sum_{|\alpha|=1}^{\infty} \underbrace{\frac{1}{\alpha !} \partial_{\bar{w}}^{\alpha} \hat{\varrho}(z(t), 0) \overline{z(t)}}_{\text {each term has factor } \bar{t}}
$$

Since the left-hand side is holomorphic in $t$ and all terms on the right hand side contain $\bar{t}$, there cannot be any non-vanishing term. Hence we get $\hat{\varrho}(z(t), 0) \equiv 0$.

\section{An immediate consequence of Lemma 2.5}

From Lemma 2.5 we can easily deduce Theorem 1.2, which was first proved in [5] as a basis for showing that for compact $X$ the set $\mathcal{A}=\emptyset$. It says that all $\mathbb{C}$-analytic curves in $X$ lie in $\mathbb{C}$-analytic subsets of "uniform size"

Proof. We are given a non-constant holomorphic curve $\gamma$ with parameterization $z(t)$ for $|t|<1$ and passing through $z$. We apply the Principal Lemma 2.5 to all points $z(t)$ on $\gamma$ instead of just the origin. We therefore get

$$
\gamma \subset V_{1}:=\bigcap_{t} S_{z(t)}
$$

However, there is an $r>0$ such that all $S_{z}$ are closed analytic hypersurfaces in polydiscs of uniform size $r$. So also $V_{1}$ is a closed analytic set in polydiscs of uniform size $r$. However the set $V_{1}$ might not be contained in $X$. Instead, we consider:

$$
A:=\bigcap_{\zeta \in V_{1}} S_{\zeta}
$$

Because of Lemma 2.5 it follows that $\gamma \subset A$. We claim that also $A \subset X$. Let for this $z \in A$ be arbitrary. Then we have

$$
z \in S_{\zeta} \quad \forall \zeta \in V_{1}
$$

according to the definition of $V_{1}$. Hence $z \in V_{1} \cap A$, which implies that $z \in S_{z}$ because of (3.1). This, however, is the case only for $z \in X$ (see Lemma 2.4(2)). Hence $A \subset X$ is, as an intersection of Segre varieties, the $\mathbb{C}$-analytic set of uniform size in $X$ containing $\gamma$ which we were looking for.

\section{Preparation of the proof of the Main Result Theorem 1.1}

In order to show that the set $\mathcal{A}$ is closed, we assume that the origin has been chosen in such a way that $0 \in \overline{\mathcal{A}}$. We have to show that $0 \in \mathcal{A}$. For this we pick a 
sequence of points $\left(a_{N}\right) \subset \mathcal{A}$ with $a_{N} \rightarrow 0$. For each $N$ we can find a non-constant holomorphic curve $\gamma_{N} \subset X$ passing through $a_{N}$. And according to Theorem 1.2 a $\mathbb{C}$-analytic set $A_{N} \subset X$ of uniform size with $\gamma_{N} \subset A_{N}$. $A_{N}$ can be chosen to be an intersection of Segre varieties (finitely many obviously suffice because of the Noether theorem):

$$
A_{N}=\bigcap_{1}^{l_{N}} S_{z_{j}^{N}} \subset X .
$$

The idea of the proof consists in trying to technically prepare the situation such that the existence of a $\mathbb{C}$-analytic limit set of positive dimension through the limit point 0 can be obtained applying Theorem 1.8 to Bishop's theorem. For this it is of course very important that we know already that the sets $A_{N}$ do not shrink down to a point, but all are closed analytic subsets of polycylinders of uniform radius. The way to achieve this will be to replace the $A_{N}$ by an analytic family of $\mathbb{C}$-analytic sets to which Theorem 1.8 can be applied in order to ensure the uniform boundedness of their volumes.

In a first step we can, after passing to a subsequence, find a sequence of points $b_{N} \in A_{N}$ and a small enough ball $B(0, r)$, such that

$$
\operatorname{dim}\left(A_{N} \cap B(0, r)\right)=\operatorname{dim}_{b_{N}}\left(A_{N}\right):=p_{N} .
$$

Next, we consider the pure dimensional $\mathbb{C}$-analytic subset

$$
B_{N}:=\left\{z \in A_{N} \mid \operatorname{dim}_{z} A_{N}=p_{N}\right\} .
$$

Since $1 \leq p_{N} \leq n$, we can pass to another subsequence of $\left(b_{N}\right)$, denoted for simplicity again by $\left(b_{N}\right)$, such that $\operatorname{dim}_{b_{N}} B_{N}=: p$ is independent of $N$. We know according to (4.1) that

$$
b_{N} \in B_{N} \subset A_{N}=\bigcap_{1}^{l_{N}} S_{z_{j}^{N}} \subset X .
$$

We now claim:

Lemma 4.1. There exists an integer $l$ such that for an open set $\tilde{U}$ and a suitable choice of l points $\left(w_{1}^{N}, \ldots, w_{l}^{N}\right) \subset\left(z_{1}^{N} \ldots, z_{l_{N}}^{N}\right)$ one has $\forall N$

$$
B_{N} \subset \bigcap_{1}^{l} S_{w_{j}^{N}}=: C_{N}
$$

with $\operatorname{dim} C_{N}=\operatorname{dim} B_{N}$ as analytic subsets of $\tilde{U}$.

Proof. We assume that $p=\operatorname{dim} B_{N}$ and let $k_{0}$ be the maximum of all integers $k$ such that there is an integer $l$ independent of $N$, and there are points $\left(w_{1}^{N}, \ldots, w_{l}^{N}\right)$ for which

$$
B_{N} \subset \bigcap_{j=1}^{l} S_{w_{j}^{N}}=C_{N}
$$


with $\operatorname{dim} C_{N}=n-k_{0}$ on some open set $\tilde{U} \subset B_{r}$. Obviously one has

$$
1 \leq k_{0} \leq n-p
$$

We make the hypothesis, that

$$
k_{0} \supsetneqq n-p
$$

and want to deduce a contradiction from it by using Theorem 1.8. By dimension theory for $\mathbb{C}$-analytic sets, we can choose generic linear combinations with complex coefficients of the holomorphic functions $\left(\hat{\varrho}\left(z, w_{1}^{N}\right) \ldots, \hat{\varrho}\left(z, w_{l}^{N}\right)\right)$ such that we have

$$
C_{N} \subset\left\{\sum_{j=1}^{l} \lambda_{j}^{1} \hat{\varrho}\left(z, w_{j}^{N}\right)=\cdots=\sum_{j=1}^{l} \lambda_{j}^{k_{0}} \hat{\varrho}\left(z, w_{j}^{N}\right)=0\right\}:=D_{N}
$$

and this representation of $D_{N}$ is a complete intersection. We apply the Theorem 1.8 of [6] to the set of functions

$$
G=\left(g_{1}, \ldots, g_{k_{0}}\right)
$$

with

$$
g_{i}:\left(z, w_{1} \cdots, w_{l}, \lambda^{1}, \cdots, \lambda^{k_{0}}\right) \rightarrow \sum_{j=1}^{l} \lambda_{j}^{i} \hat{\varrho}\left(z, w_{j}\right)
$$

where we consider the $w_{j}$ and all coefficients $\lambda_{j}^{i}$ as complex parameters. The $g_{j}$ are holomorphic in these variables (literally speaking they are, of course, antiholomorphic in the $w^{\prime} s$. This, however, does not harm at all). Since (4.6) is a complete intersection, and therefore pure-dimensional, Theorem 1.8 anyhow applies and we obtain for any open subset $U \Subset \tilde{U}$ a constant $c(U)$, such that

$$
\operatorname{vol}_{2\left(n-k_{0}\right)}\left(D_{N}\right) \leq c(U) .
$$

Hence, in any relatively compact subset of $\tilde{U}$ the number of irreducible components of $C_{N}$ of dimension $n-k_{0}$ is uniformly bounded with respect to $N$. We, therefore, can find a number $l^{\prime}$ and another family of points $\left(\theta_{1}^{N}, \ldots, \theta_{l^{\prime}}^{N}\right)$ among the points $\left(z_{1}^{N}, \ldots, z_{l_{N}}^{N}\right)$ such that

$$
B_{N} \subset C_{N} \cap\left\{\hat{\varrho}\left(z, \theta_{1}^{N}\right)=\cdots=\hat{\varrho}\left(z, \theta_{l^{\prime}}^{N}\right)=0\right\}:=D_{N}^{\prime}
$$

with

$$
\operatorname{dim} D_{N}^{\prime} \supsetneqq n-k_{0} \quad \forall N .
$$

Notice that the number $l^{\prime}$ depends on $k_{0}$ only and the number of $\left(n-k_{0}\right)$-dimensional irreducible components of $C_{N}$ and, therefore, is independent of $N$.

This is the desired contradiction to the maximality of $k_{0}$. 


\title{
5. End of the proof of Theorem 1.1
}

We, now can finish the proof of Theorem 1.1 by again applying the Theorem 1.8 of [6] to the functions

$$
\begin{gathered}
G: \tilde{U} \times\left(B_{r+\varepsilon}\right)^{l} \times \mathbb{C}^{l(n-p)} \rightarrow \mathbb{C}^{n-p} \\
\left(z, w_{1}, \cdots, w_{l}, \lambda^{1}, \cdots, \lambda^{n-p}\right) \rightarrow\left(\sum_{j=1}^{l} \lambda_{j}^{1} \hat{\varrho}\left(z, w_{j}\right), \cdots, \sum_{j=1}^{l} \lambda_{j}^{n-p} \hat{\varrho}\left(z, w_{j}\right)\right) .
\end{gathered}
$$

We get $\operatorname{vol}_{2 p}\left(B_{N}\right) \leq c(U) \forall U \Subset \tilde{U}$. Hence we get from the theorem of Bishop that there is a $\mathbb{C}$-analytic limit set $A$ of a suitable subsequence of $\left(B_{N}\right)$. It satisfies $\operatorname{dim} A=p>0$ and $0 \in A \subset X$. Hence $0 \in \mathcal{A}$, as we wanted to show.

\section{References}

[1] E. BISHOP, Condition for the analyticity of certain sets, Michigan Math. J. 11 (1964), 289304.

[2] E. M. ChIR KA, “Complex Analytic Sets”, Kluwer, Dordrecht, 1990.

[3] J. D' Angelo Real hypersurfaces, orders of contact, and applications, Ann. of Math. 115 (1982), 615-637.

[4] J. P. D'Angelo, "Several Complex Variables and the Geometry of Real Hypersurfaces", CRC Press, Boca Raton, FL, 1993.

[5] K. DIEDERICH and J. E. FORNÆSS, Pseudoconvex domains with real analytic boundary, Ann. of Math. 107 (1978), 371-384.

[6] K. DIEDERICH and S. PINCHUK, Uniform volume estimates for holomorphic families of analytic sets, Proc. Steklov Inst. Math., 235 (2001), 52-56.

[7] J. KoHn, Subellipticity of the $\bar{\partial}-$ Neumann problem on pseudoconvex domains: Sufficient conditions, Acta. Math. 142 (1979), 79-122.

[8] E. MAZZILLI, Germes d'ensembles analytiques dans une hypersurface algébrique, Ark. Mat. 44 (2006), 327-333.

\author{
Mathematik \\ Universität Wuppertal \\ Gausstr. 20 \\ D-42097 Wuppertal, Germany \\ klas@uni.wuppertal.de \\ Département de Mathématiques \\ CNRS URA D751 \\ Université Lille 1 \\ F-59655 Villeneuve d'Ascq, France \\ emmanuel.mazzilli@agat.univ-lille1.fr
}

